\title{
Simulation Meets Experiment: Unraveling the Properties of Water in Metal-Organic Frameworks Through Vibrational Spectroscopy
}

Kelly M. Hunter, *† Jackson C. Wagner, † Mark Kalaj, † Seth Cohen, †キ Wei Xiong, *†‡ Francesco Paesani $* \dagger \neq \mathbb{T}$

† Department of Chemistry and Biochemistry, University of California San Diego, La Jolla, California 92093, United States

*†キ Materials Science and Engineering, University of California San Diego, La Jolla, California 92093, United States

*†キ San Diego Supercomputer Center, University of California San Diego, La Jolla, California 92093, United States

*E-mails:k6hunter@ucsd.edu,w2xiong@ucsd.edu,fpaesani@ucsd.edu 


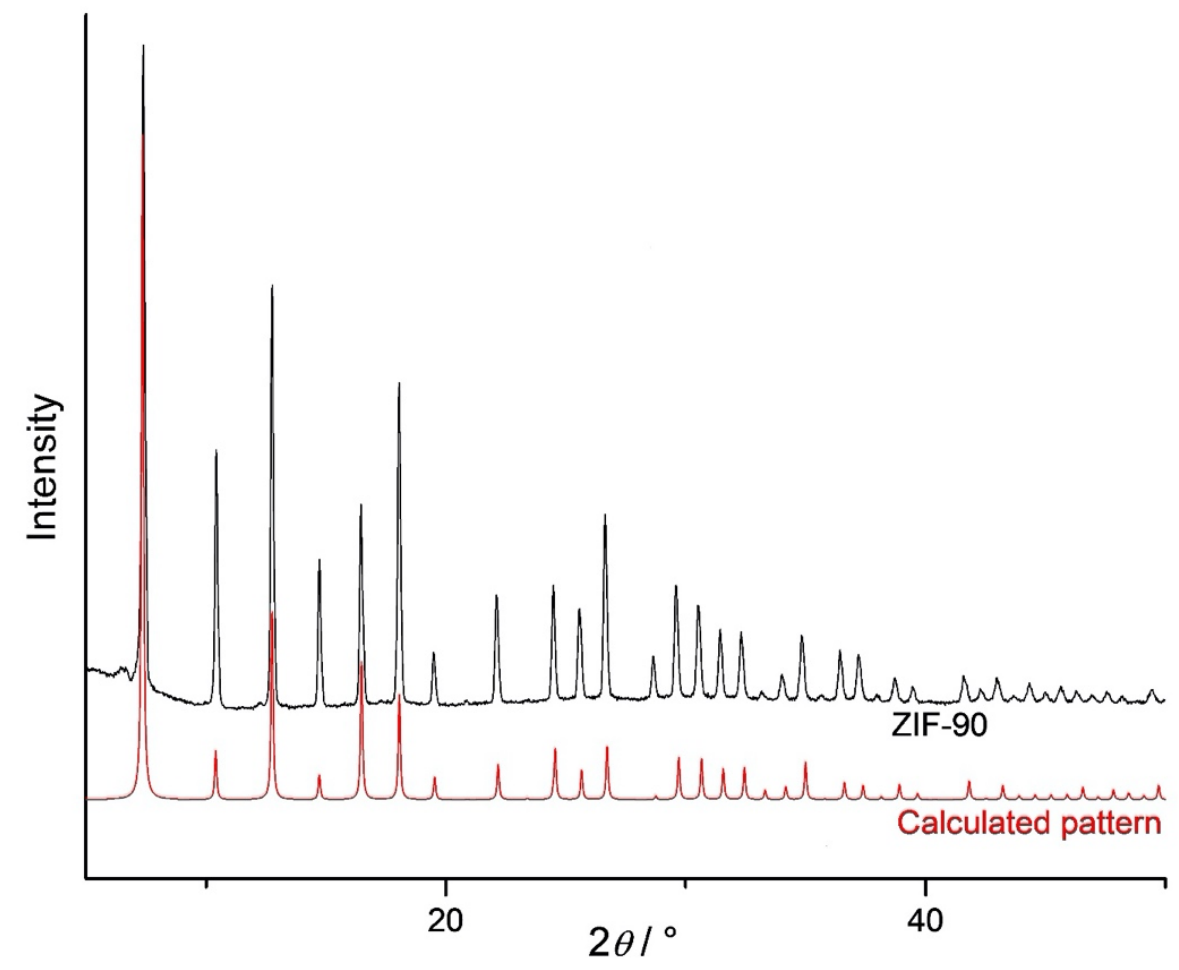

Figure S1: As synthesized ZIF-90 PXRD (black) and calculated PXRD spectra of ZIF-90 (red).

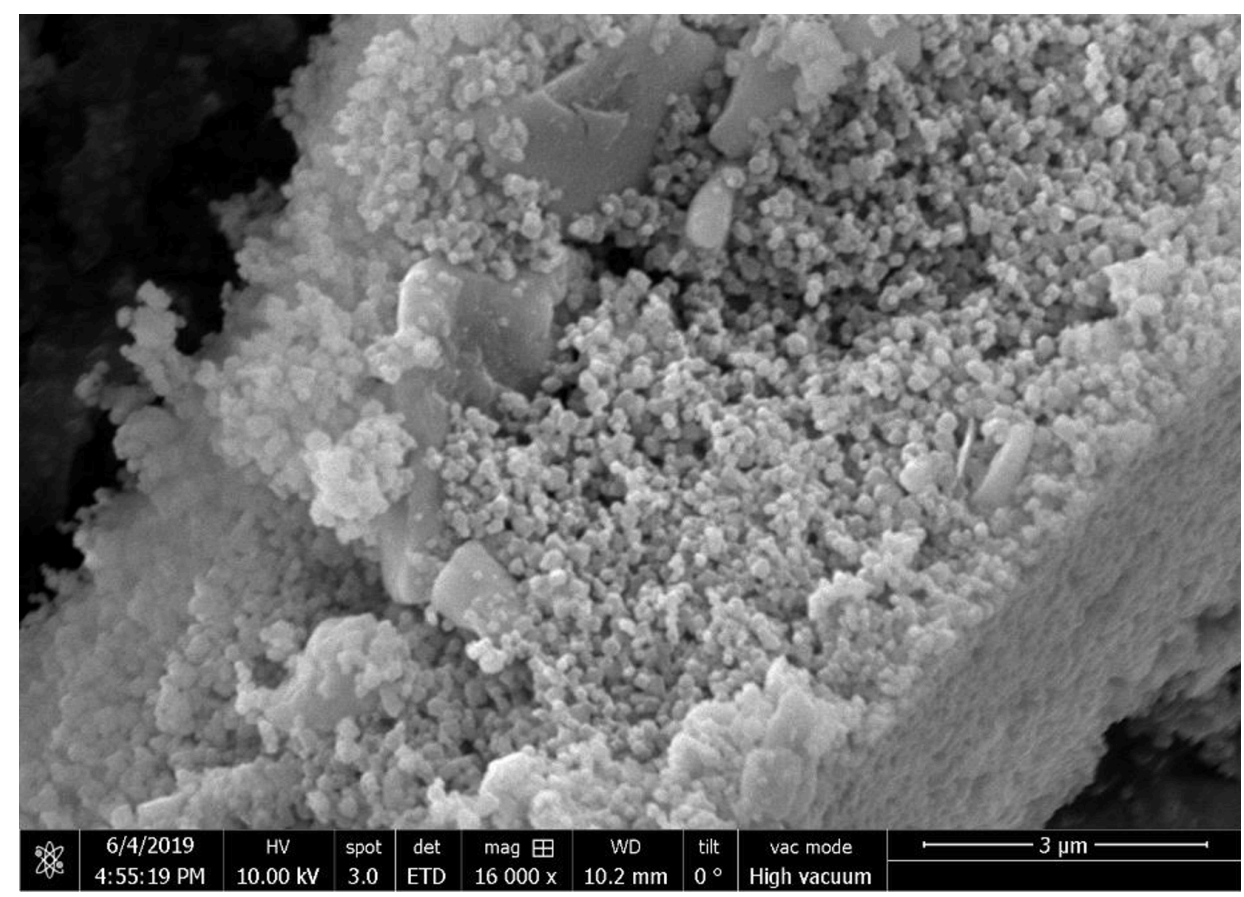

Figure S2: SEM image of nanoscale synthesis of ZIF-90. 


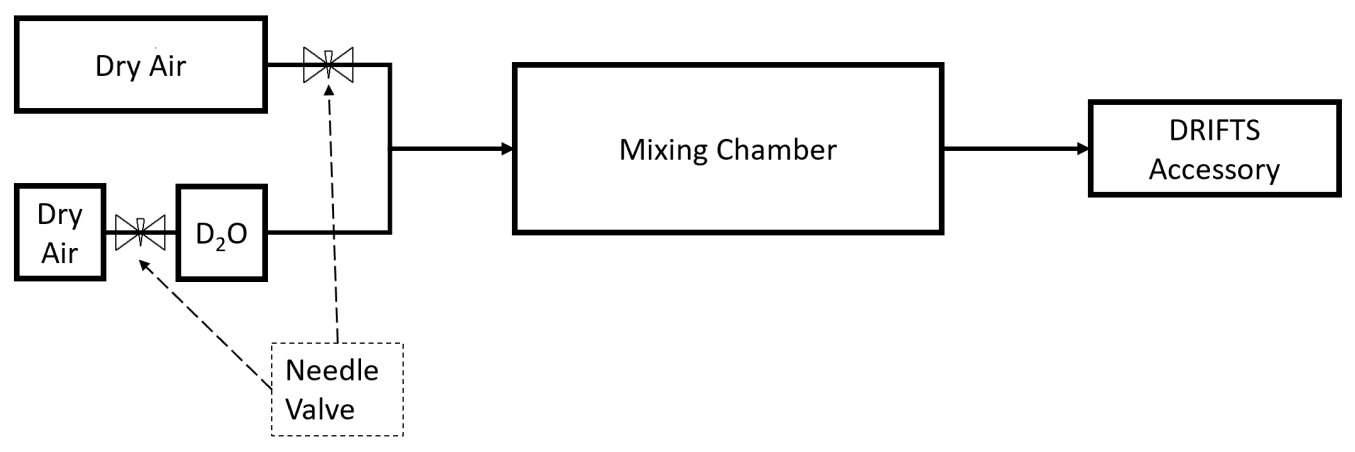

Figure S3: Humidity Generator schematic. Humidified air was generated by mixing differing amounts of dry air and dry air bubbled through $\mathrm{D}_{2} \mathrm{O}$. The flow of each dry air gas stream was manually controlled with needle valves.

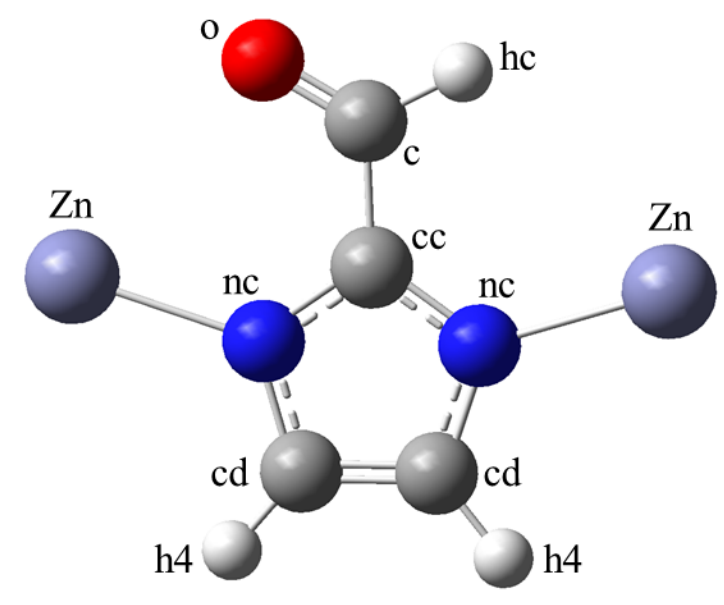

Figure S4: Atom labels used in the definition of the force field parameters for ZIF-90. 
The complete list of parameters of the force field for ZIF-90 is reported in Tables 1-4.

Table S1. Force field parameters for ZIF-90. Electrostatic and Lennard-Jones potentials.

\begin{tabular}{ccccc}
\hline Atom name & Atom type & Charge & $\varepsilon\left(\mathrm{kcal} \cdot \mathrm{mol}^{-1}\right)$ & $\sigma / 2(\AA)$ \\
\hline $\mathrm{Zn}$ & $\mathrm{Zn}$ & 0.6428 & 0.01251 & 0.98000 \\
$\mathrm{nc}$ & $\mathrm{nc}$ & -0.2993 & 0.17010 & 1.62500 \\
$\mathrm{o}$ & $\mathrm{o}$ & -0.3475 & 0.21000 & 1.66120 \\
$\mathrm{c}$ & $\mathrm{c}$ & 0.1864 & 0.08605 & 1.70000 \\
$\mathrm{cc}$ & $\mathrm{cc}$ & 0.1937 & 0.08605 & 1.70000 \\
$\mathrm{~cd}$ & $\mathrm{~cd}$ & -0.0062 & 0.08605 & 1.70000 \\
$\mathrm{~h} 4$ & $\mathrm{~h} 4$ & 0.1059 & 0.01501 & 1.25500 \\
$\mathrm{hc}$ & $\mathrm{hc}$ & 0.0452 & 0.01501 & 1.25500 \\
\hline
\end{tabular}

Table S2. Force field parameters for ZIF-90. Bond potentials: Harmonic functions.

\begin{tabular}{ccc}
\hline Bond type (Harmonic) & $\mathrm{K}_{i j}\left(\mathrm{kcal} \mathrm{mol}^{-1} \AA^{2}\right)$ & $r_{0}(\AA)$ \\
\hline Zn-nc & 74.50 & 2.024 \\
nc-cc & 441.1 & 1.369 \\
nc-cd & 525.4 & 1.317 \\
cc-c & 371.0 & 1.468 \\
c-hc & 310.7 & 1.112 \\
c-o & 637.7 & 1.218 \\
cd-cd & 419.8 & 1.428 \\
cd-h4 & 325.0 & 1.082 \\
\hline
\end{tabular}

Table S3. Force field parameters for ZIF-90. Bending potentials.

\begin{tabular}{ccc}
\hline Angle type (Harmonic) & $\mathrm{K}_{i j k}\left(\mathrm{kcal} \mathrm{mol}^{-1} \mathrm{rad}^{2}\right)$ & $\theta\left(^{\circ}\right)$ \\
\hline Zn-nc-cc & 14.43 & 126.8500 \\
Zn-nc-cd & 11.36 & 126.9500 \\
nc-Zn-nc & 11.09 & 109.4200 \\
nc-cc-nc & 69.80 & 125.7001 \\
nc-cc-c & 66.20 & 123.3201 \\
nc-cd-cd & 71.60 & 112.5600 \\
nc-cd-h4 & 52.10 & 118.4701 \\
cc-nc-cd & 71.80 & 105.4900 \\
cc-c-hc & 47.10 & 114.8300 \\
cc-c-o & 69.10 & 123.9301 \\
hc-c-o & 54.20 & 120.7001 \\
cd-cd-h4 & 45.90 & 127.9601 \\
\hline
\end{tabular}


Table S4. Force field parameters for ZIF-90. Torsion potentials.

\begin{tabular}{cccc}
\hline Dihedral type & $\mathrm{K}_{i j k}\left(\mathrm{kcal} \mathrm{mol}^{-1}\right)$ & $\chi\left(^{\circ}\right)$ & $N$ \\
\hline Zn-nc-cd-h4 & 1.0560 & 180.000 & 2 \\
Zn-nc-cd-cd & 1.4160 & 180.000 & 2 \\
Zn-nc-cc-c & 0.2270 & 180.000 & 2 \\
Zn-nc-cc-nc & 0.6140 & 180.000 & 3 \\
nc-Zn-nc-cc & 0.0260 & 0.000 & 3 \\
nc-Zn-nc-cd & 0.0210 & 180.000 & 2 \\
nc-cc-nc-cd & 4.7500 & 180.000 & 2 \\
nc-cc-c-hc & 2.8750 & 180.000 & 2 \\
nc-cc-c-o & 2.8750 & 180.000 & 2 \\
nc-cd-cd-nc & 4.0000 & 180.000 & 2 \\
nc-cd-cd-h4 & 4.0000 & 180.000 & 2 \\
cc-nc-cd-cd & 4.7500 & 180.000 & 2 \\
cc-nc-cd-h4 & 4.7500 & 180.000 & 2 \\
c-cc-nc-cd & 4.7500 & 180.000 & 2 \\
h4-cd-cd-h4 & 4.0000 & 180.000 & 2 \\
nc-Zn-cc-cd & 0.0560 & 180.000 & 2 \\
cc-nc-c-nc & 1.1000 & 180.000 & 2 \\
c-hc-cc-o & 10.500 & 180.000 & 2 \\
cd-h4-cd-nc & 1.1000 & 180.000 & \\
\hline
\end{tabular}

Table S5. Average simulation box sizes for various RH. NPT simulations were performed for 500 ps at each RH.

\begin{tabular}{cc}
\hline$\% \mathrm{RH}$ & Average box dimension $(\AA)$ \\
\hline Experiment & 17.27150 \\
$25 \%$ & 17.44905 \\
$30 \%$ & 17.44765 \\
$35 \%$ & 17.39490 \\
$40 \%$ & 17.34805 \\
$45 \%$ & 17.34730 \\
$50 \%$ & 17.34525 \\
$60 \%$ & 17.34060 \\
$70 \%$ & 17.34855
\end{tabular}



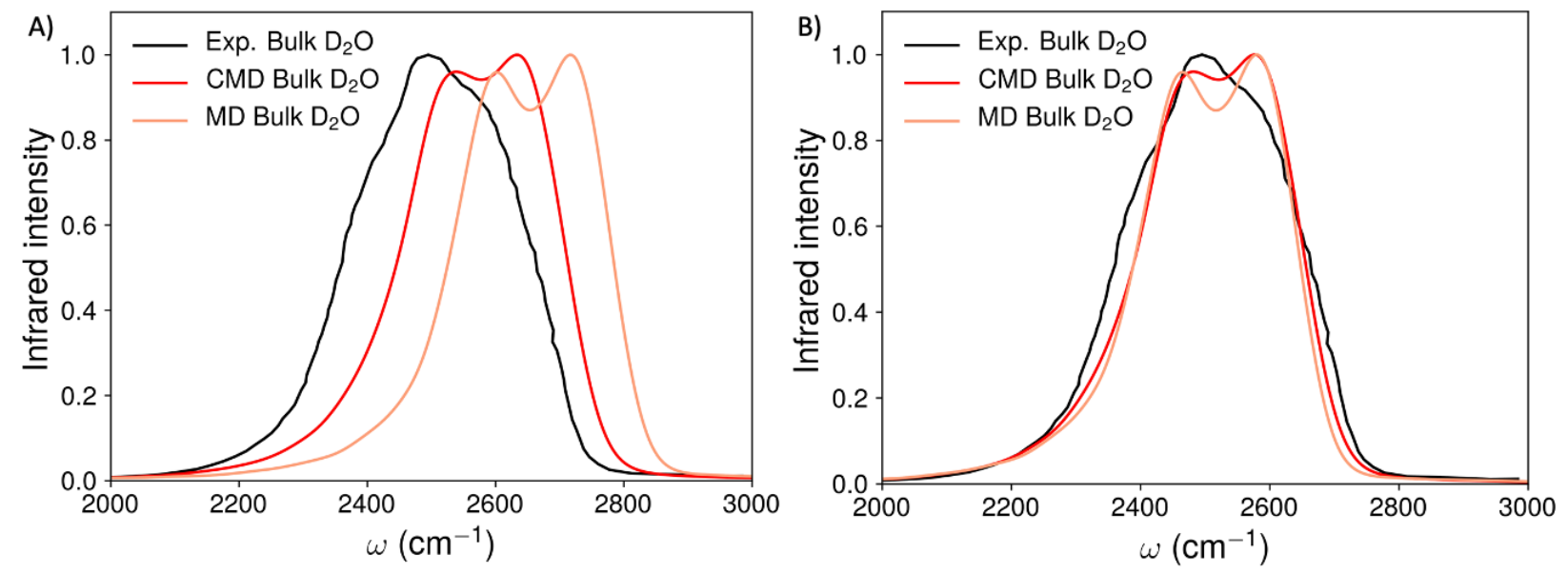

Figure S5: A) Infrared spectrum of experimental bulk $\mathrm{D}_{2} \mathrm{O}$ (black), centroid molecular dynamics (CMD) bulk $\mathrm{D}_{2} \mathrm{O}$ spectrum (red), and molecular dynamics (MD) bulk $\mathrm{D}_{2} \mathrm{O}$ spectrum (light red). Spectra are unshifted; there is a $57 \mathrm{~cm}^{-1}$ difference between CMD and experiment, and there is a $80 \mathrm{~cm}^{-1}$ difference between CMD and MD. B) Same as panel A, except the CMD spectrum (red) is red-shifted by $57 \mathrm{~cm}^{-1}$, and the MD spectrum (light red) is red-shifted by $137 \mathrm{~cm}^{-1}\left(80 \mathrm{~cm}^{-1}\right.$ for $\mathrm{MD}$ to $\mathrm{CMD}+57 \mathrm{~cm}^{-1}$ for $\mathrm{CMD}$ to experiment) All spectra are normalized to one.

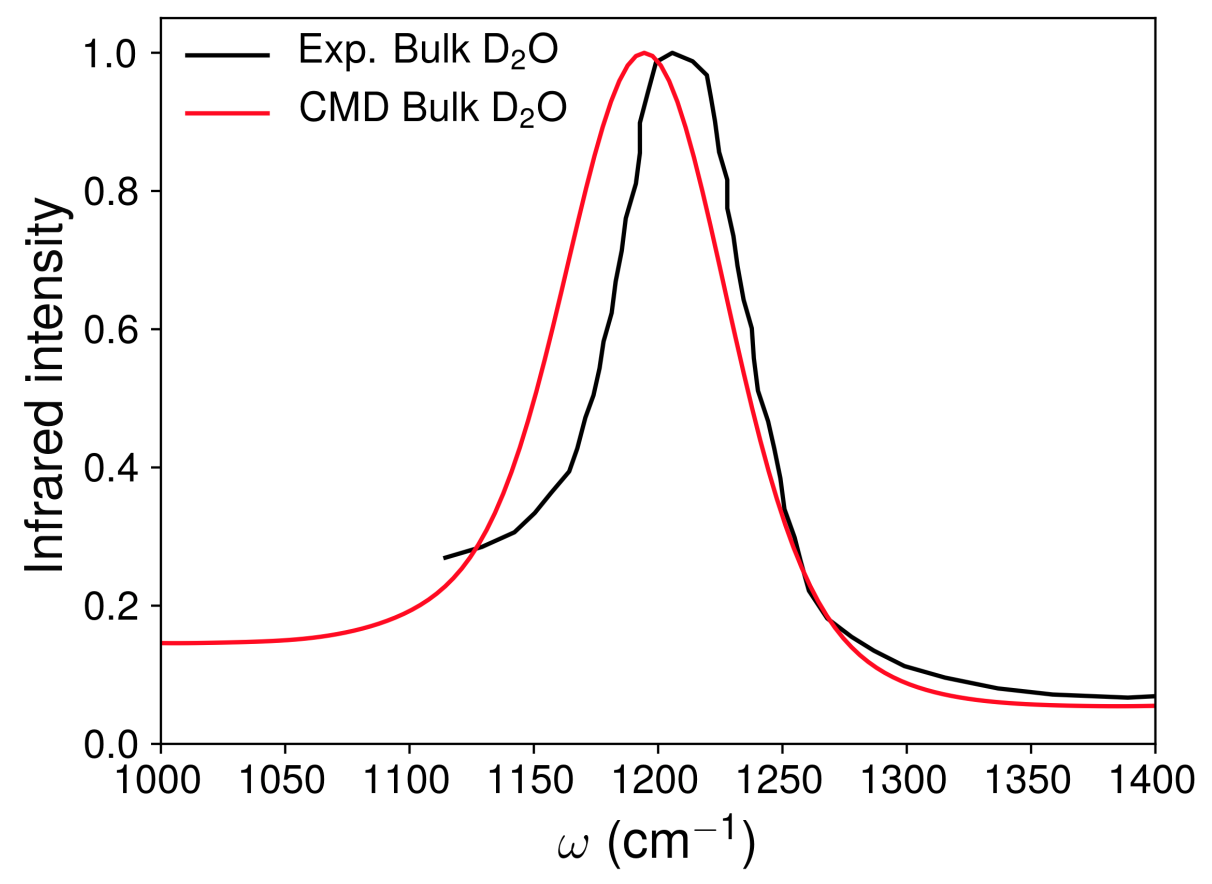

Figure S6: Experimental bulk $\mathrm{D}_{2} \mathrm{O}$ spectrum (black) and $\mathrm{CMD}$ bulk $\mathrm{D}_{2} \mathrm{O}$ spectrum (red) in the bending region. There is a $12 \mathrm{~cm}^{-1}$ red-shift of the simulated spectrum compared to the experimental spectrum. Therefore, the calculated Fermi resonance was blue-shifted by $24 \mathrm{~cm}^{-1}$ in the OD-stretching region. 


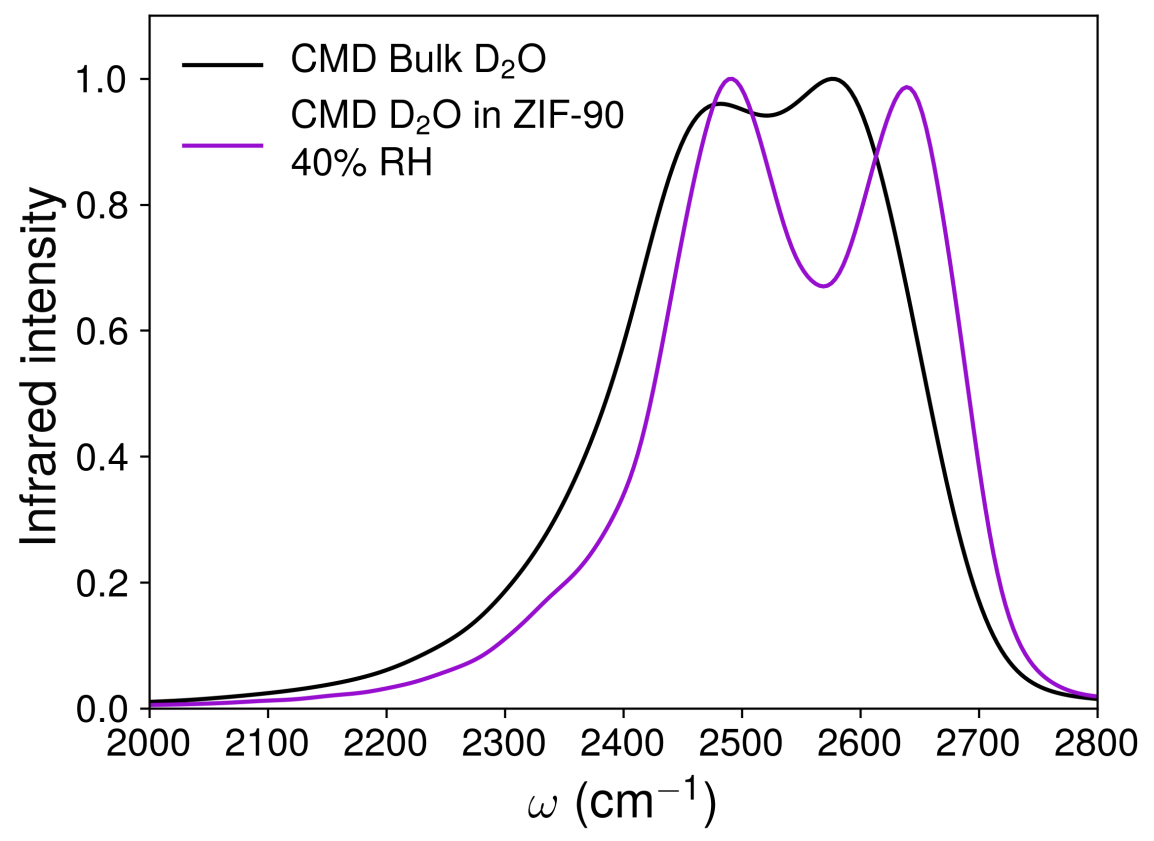

Figure S7: CMD bulk $\mathrm{D}_{2} \mathrm{O}$ spectrum (black) and CMD spectrum of $\mathrm{D}_{2} \mathrm{O}$ in $\mathrm{ZIF}-90$ at $40 \% \mathrm{RH}$ (purple) displaying the blue-shift in going from bulk water to confined water.
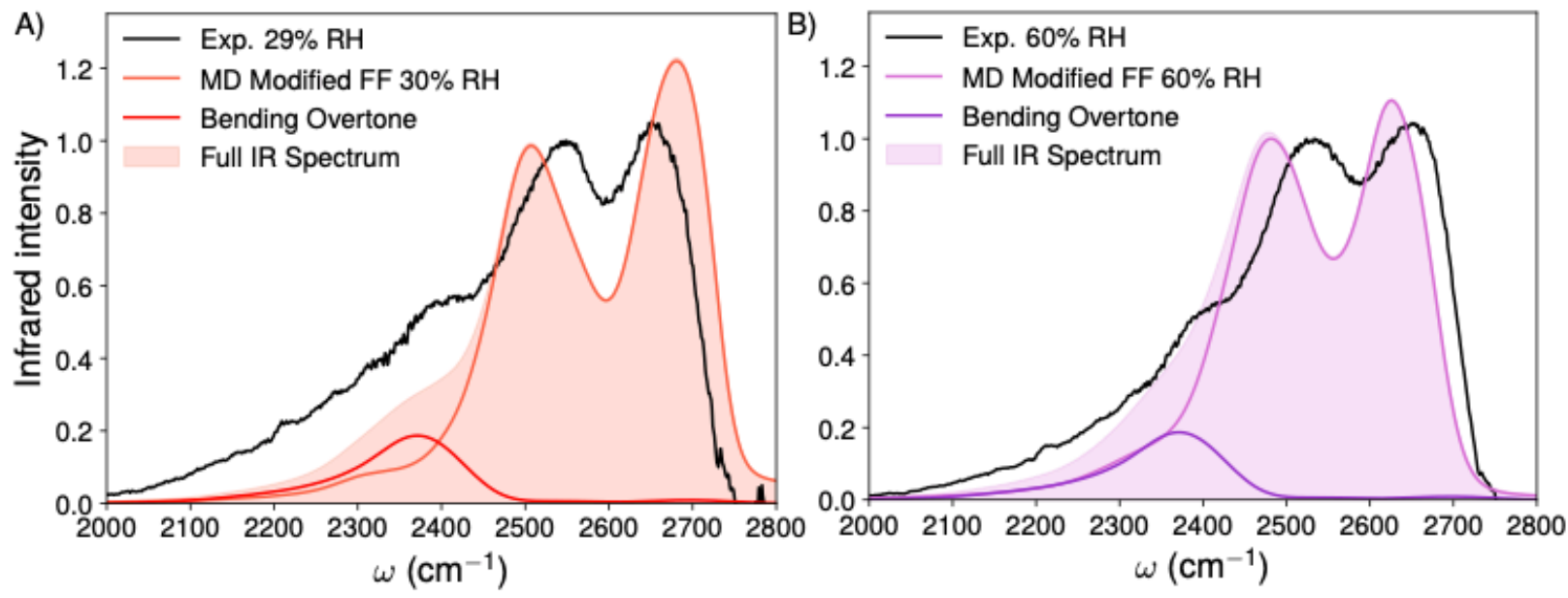

Figure S8: A) Experimental IR spectrum of water in ZIF-90 at 29\% RH (black), MD 30\% RH spectrum (pink), Fermi resonance contribution (red), and MD 30\% RH spectrum with added Fermi resonance contribution (shaded pink). B) Experimental IR spectrum of water in ZIF-90 at $60 \%$ RH (black), MD 60\% RH spectrum (light purple), Fermi resonance contribution (dark purple), and MD $60 \%$ RH spectrum with added Fermi resonance contribution (shaded light purple). All theoretical spectra are red-shifted by $137 \mathrm{~cm}^{-1}$, DOD overtones are blue-shifted by $24 \mathrm{~cm}^{-1}$, and all spectra are normalized to one at the lower frequency peak. 


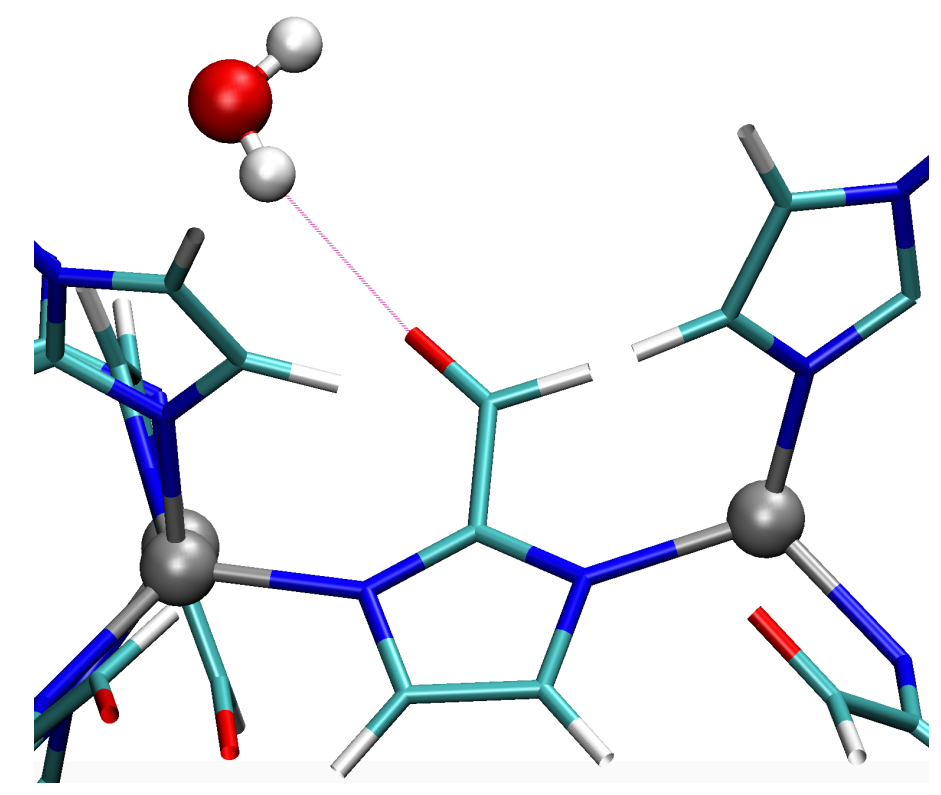

Figure S9: Water forming a H-bond to the aldehyde group of the organic linker of ZIF-90.

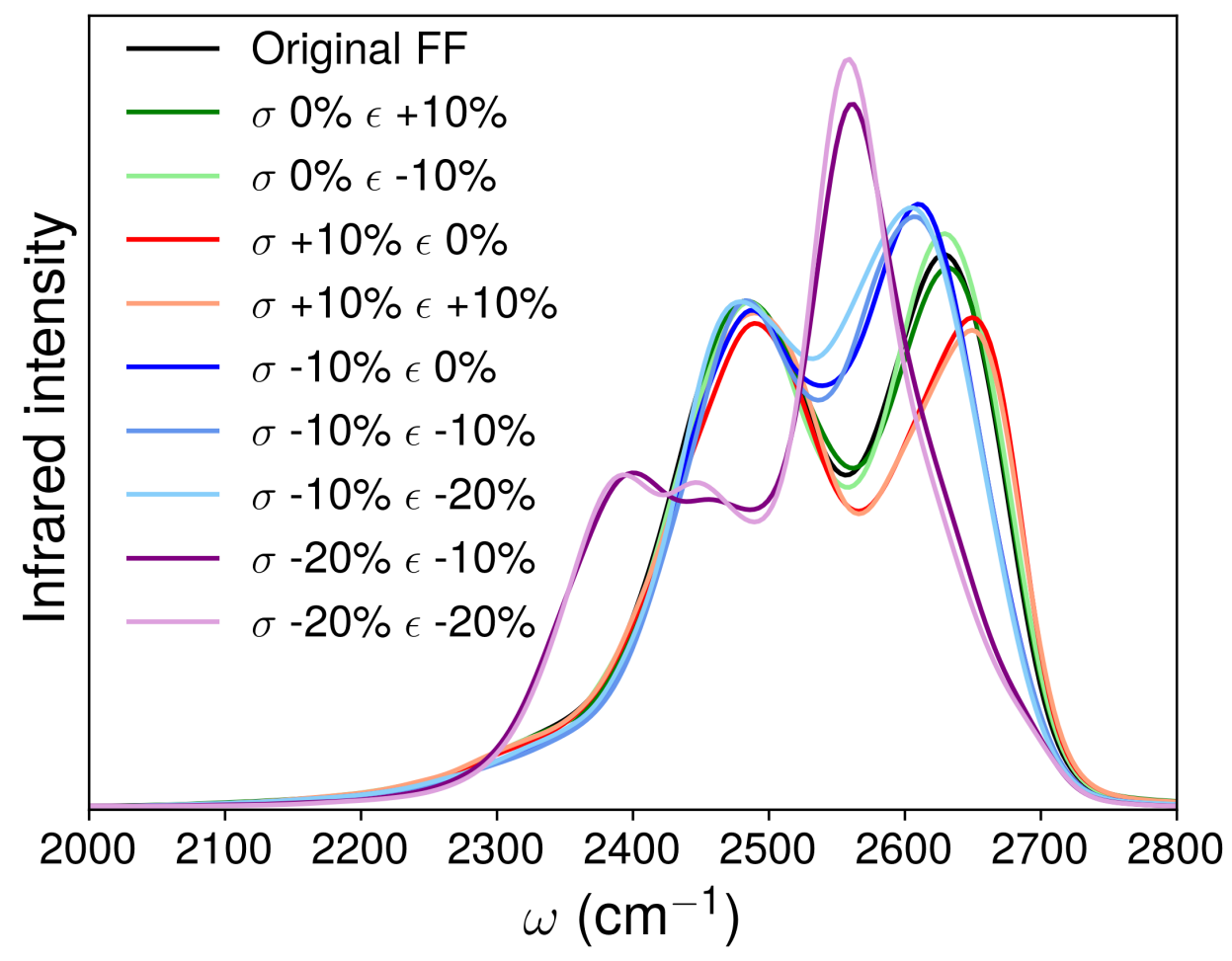

Figure S10: Many-body IR spectra at $40 \% \mathrm{RH}$ varying the LJ interactions of water with every MOF atom. $\sigma$ and $\epsilon$ are increased or decreased by the percent listed: $0 \%$ means the value is unchanged from the original FF, a positive value means the interaction was increased from the original FF, and a negative value means the interaction was decreased from the original FF. All spectra are red-shifted by $137 \mathrm{~cm}^{-1}$. 

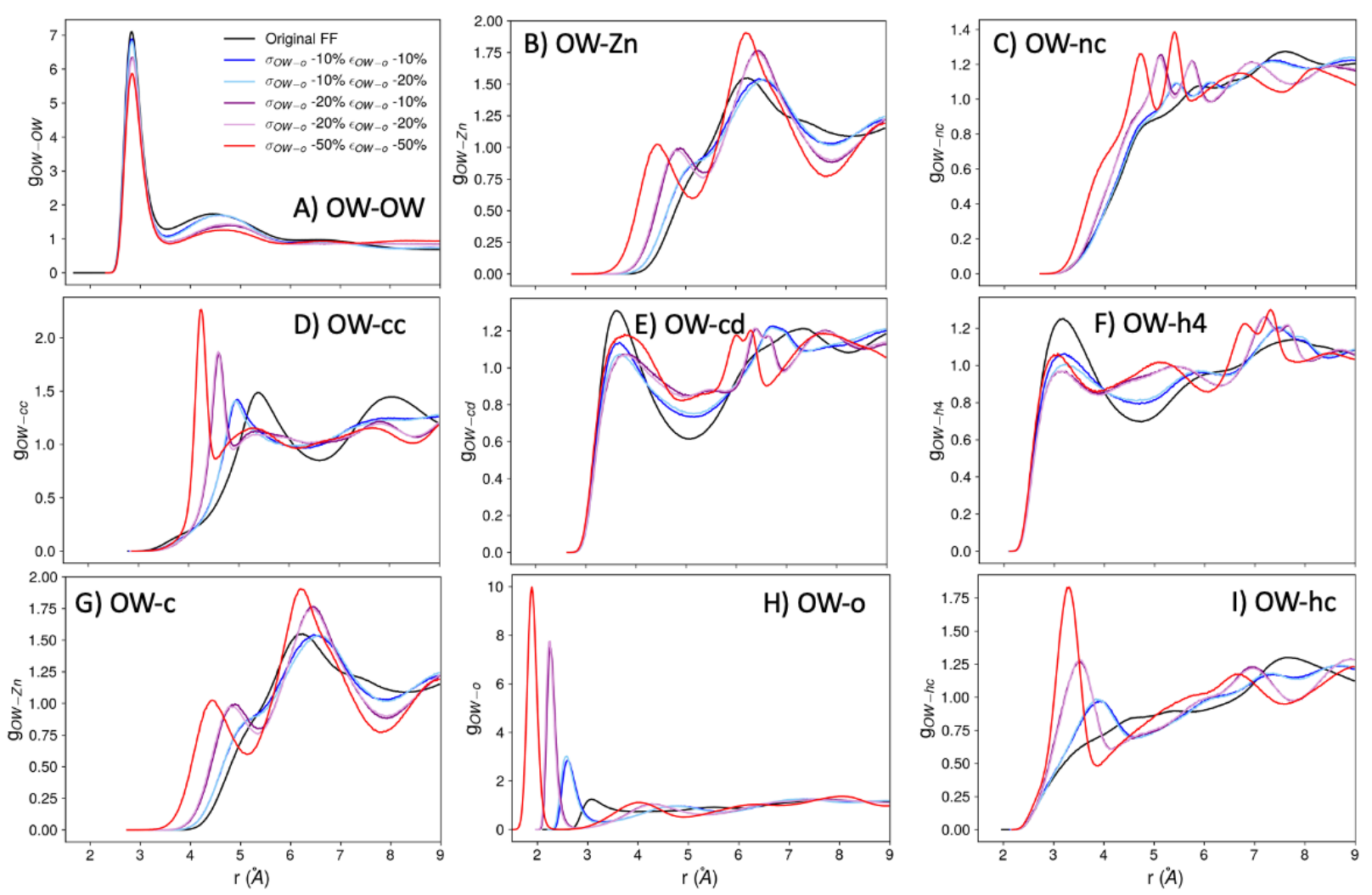

Figure S11: Radial distribution functions (RDFs) of the OW water atom confined in ZIF-90 for the simulations changing the OW-o LJ parameters. RDFs are plotted by the value of $\sigma$ : the original force field (black), a decrease of $10 \%$ for $\sigma$ (blue), a decrease of $20 \%$ for $\sigma$ (purple), and a decrease of $50 \%$ for $\sigma$ (red). MOF atom labels are given in Figure S4. 

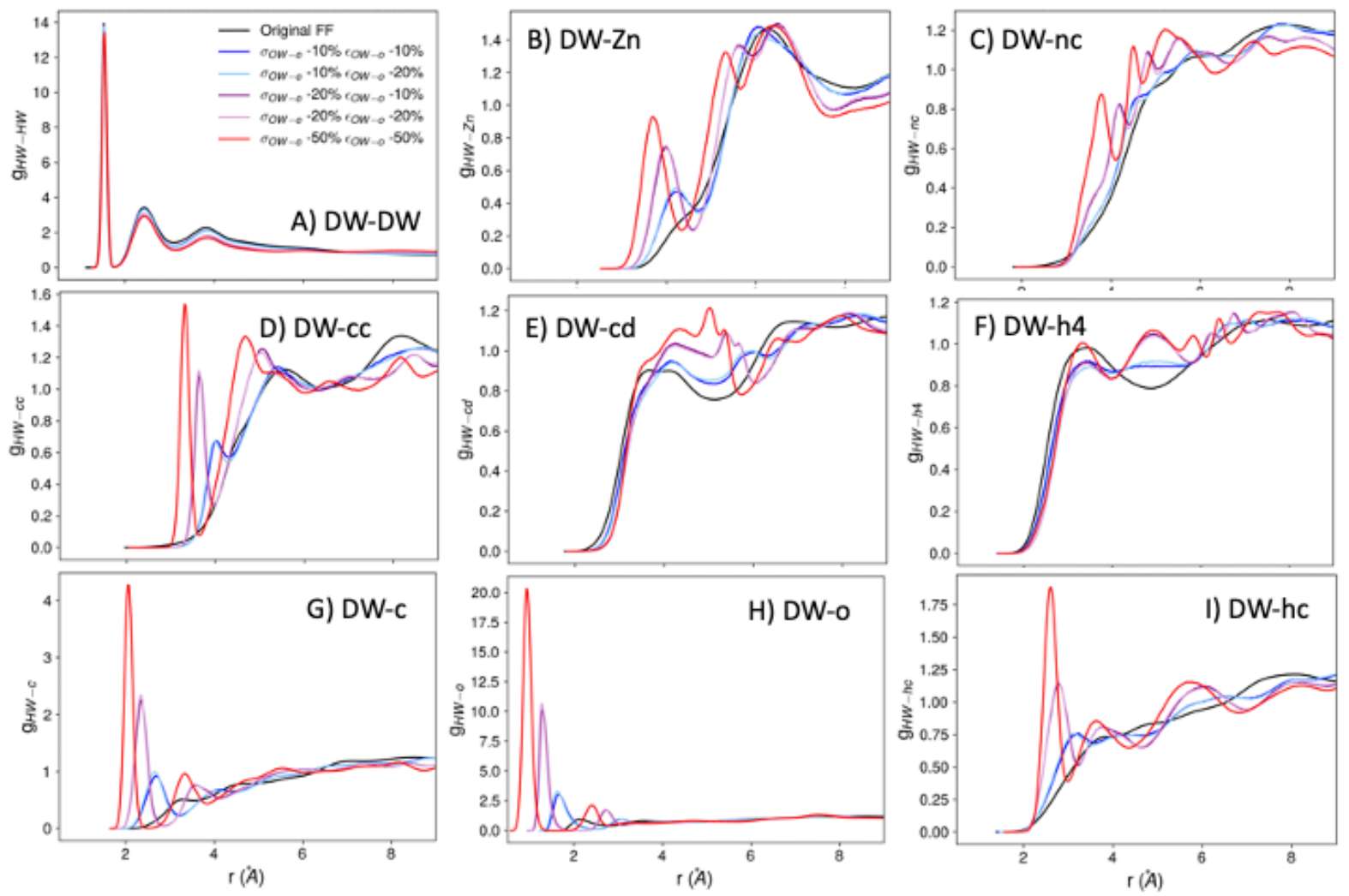

Figure S12: Radial distribution functions (RDFs) of the DW water atom confined in ZIF-90 for the simulations changing the OW-o LJ parameters. RDFs are plotted by the value of $\sigma$ : the original force field (black), a decrease of $10 \%$ for $\sigma$ (blue), a decrease of $20 \%$ for $\sigma$ (purple), and a decrease of $50 \%$ for $\sigma$ (red). MOF atom labels are given in Figure S4.
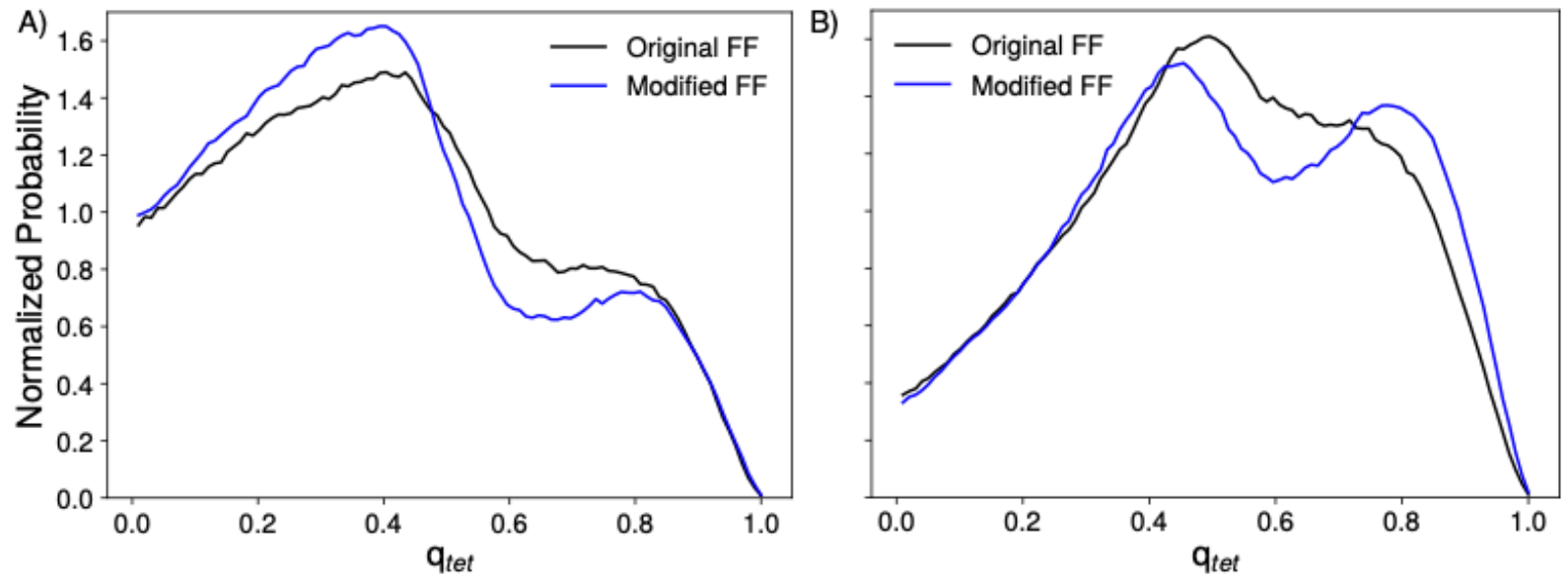

Figure S13: Tetrahedral order parameters of A) only water molecules and B) water molecules plus the o atom of the carbonyl group of the framework for the original force field (FF, black) and modified force field decreasing $\sigma$ by $10 \%$ and $\epsilon$ by $20 \%$ (blue). 

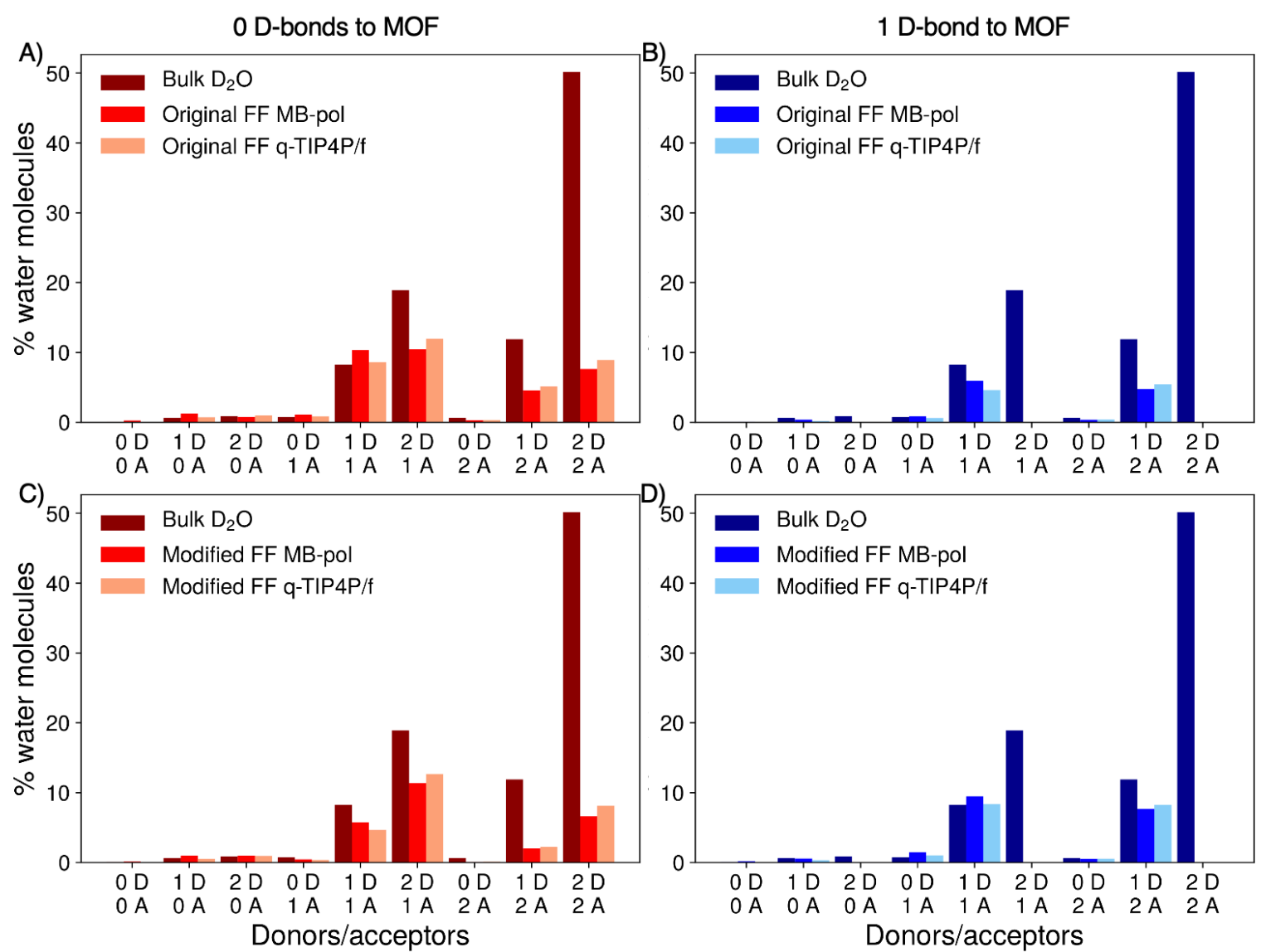

Figure S14: D-bonding topology of the original (top row) and modified (bottom row) force fields using MB-pol (darker colors) and q-TIP4P/f (lighter colors) at 40\% RH with MB-pol bulk water (darkest colors). Percent of water molecules donating (D) and accepting (A) D-bonds shown when $0 \mathrm{D}$-bonds are donated to the MOF (left column) and when $1 \mathrm{D}$-bond is donated to the MOF (right column). 